\title{
A New UK Old Age Psychiatry Curriculum is Needed
}

\author{
V M Aziz ${ }^{1 *}$ and Rugiyya Saeed ${ }^{2}$ \\ ${ }^{1}$ Consultant Psychiatrist, UK \\ ${ }^{2}$ Specialty Trainee in Old Age Psychiatry, ,UK \\ *Corresponding author: VM Aziz, Consultant Psychiatrist, Cynon Valley Hospital, New Road, Mountain Ash, UK
}

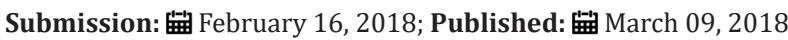

\begin{abstract}
This article looks at the current training, the need for new old age psychiatry curriculum in the UK and the drivers for this. Keywords: Curriculum; Old age psychiatry; Higher training
\end{abstract}

Key Points

1. The current old age psychiatry curriculum was developed in 2010 and updated in 2012.

2. The generic professional capabilities (GPC) aims to identify, simplify and clarify the important core professional capabilities doctors should possess at specialist registration.

3. Supervised learning experiences (SLE) will replace WPBA.

4. There is a need to review the current advanced curriculum for old age psychiatry in the UK.

\section{Mini Review}

The higher training programme in psychiatry is comprised of completion of a minimum of 36 months of advanced training from ST4 to ST6 in one of the six GMC approved psychiatric specialties, one of which is old age psychiatry. Trainee doctors enter the higher training programme in their chosen specialty once they have completed core training and passed the MRCP sych examinations. The advanced curriculum provides a framework during higher training and is specific for the chosen specialty. It outlines the competencies trainees must demonstrate via work-place based assessment (WPBA), reflections and case logs to achieve an ARCP outcome 6 and be awarded a Certificate of Completion of Training (CCT), and subsequently be able to practice as a consultant psychiatrist in the UK.

The current old age psychiatry curriculum was developed in 2010 and updated in 2012. During this period, the GMC published guidance on 'Treatment and care towards the end of life: good practice in decision making' in 2010 [1], and 'Leadership and management for all doctors' in 2012 [2]. Incorporating them, the Old Ag Faculty produced its new criteria in 2014 that was approved by the College and the GMC after extensive consultations.

From 1 July to 27 September 2015, the GMC and Academy of Medical Royal Colleges consulted jointly on a draft framework for generic professional capabilities (GPC). The nine-domain framework [3] aims to identify, simplify and clarify the important core professional capabilities doctors should possess at specialist registration. GPCs will act as an indicative curriculum framework and will for the first time explicitly state educational outcomes required of all postgraduate medical curricula across the 66 medical specialties and 32 sub-specialties in the UK. It will also ensure that as requested by the service and the government that common capabilities are addressed consistently across the medical workforce. Now the College has been asked to map the psychiatry core and higher training curricula to the new capabilities.

Another change to the current advanced curriculum is going to be the introduction of supervised learning experiences (SLE), which are soon going to replace the old WPBA system. SLEs are not assessments, but an interaction between a trainee doctor and a trainer, which leads to immediate feedback and reflective learning. They are designed to help trainee doctors develop and improve their clinical and professional practice, and to set targets for future achievements. The topics covered by SLE should reflect and map the curriculum, and the aim would be choosing topics that the trainee doctor finds challenging, or an area they wish to improve upon. It would be the trainee doctor's responsibility to arrange an appropriate range as well as the required number of SLEs. The clinical supervisor's end of placement reports will draw upon evidence of engagement in the SLE process, but not the SLE outcomes.

2016 was a busy year with numerous developments in old age psychiatry. During this year, the GMC approved the new liaison 
curriculum in both adult and old age psychiatry and asked the College to map all curricula to the GMC's 'Good Medical Practice'. Also, the Royal College of Psychiatrists set up the Gatsby/Wellcome Neuroscience Project Implementation Group [4] to ensure that neuroscience is reflected in the curriculum and examination syllabus. As old age psychiatrists work closely with neurologists and neuropsychiatrists, there is a need for our higher trainees to understand neuroscience. Although it is important to acknowledge that an increasing focus and developments in this area may lead to increased recruitment of potential old age psychiatrists into being neuropsychiatrists.

Additionally, 2016 saw the publication of the OP100 report [5] 'improving the physical health of adults with severe mental illness: essential actions', which highlighted the need for the same level of physical healthcare for people with mental illnesses as that available for the general population.

The close working between the Old Age Faculty and the British Geriatric Society (BGS) in 2016 highlighted the need for a more integrated curriculum which includes training around physical health care and end of life care for psychiatry trainees, and a focus on psychiatric morbidity for the BGS trainees. When considering patient care in psychiatry, mental health comes hand in hand with the physical health of patients, particularly in old age psychiatry where the majority of the older adult population have several comorbidities. However, post MMC most psychiatry trainees have entered ST4 with only 4-8 months worth of medical specialty exposure during their foundation training. Hence there is a need for this to be addressed by the training curriculum.

Currently the specialty training curriculum for old age psychiatry only briefly mentions physical healthcare, which is tagged onto a learning outcome about clinical assessment that addresses the need for a physical assessment as part of the holistic approach to patient care. The consensus amongst trainee doctors is that they would encourage a change in the training system that would allow for more exposure to other clinical areas, both in the community and hospital settings. This could include acute outpatients or community interface with specialist services in geriatric medicine as well as developing joint training events locally or nationally. This could also make the training programme more attractive and hence improve recruitment and retention in old age psychiatry.

\section{Conclusion}

There is a need to review the current advanced curriculum for old age psychiatry in the UK going forward. Recent reports have highlighted the need for psychiatrists to improve their knowledge and skills in other clinical areas in order to deliver a holistic approach to patient health care. Particularly for old age psychiatry trainee doctors, training around physical health and end of life care is valuable; this is a need that has been identified by the current trainee doctors.

\section{References}

1. The General Medical Council (2010) Treatment and care towards the end of life: good practice in decision making.

2. The General Medical Council (2012) Leadership and management for all doctors.

3. The General Medical Council (2016) Framework for generic professional capabilities (GPCs).

4. The Royal College of Psychiatrist (2016) The gatsby/wellcome neuroscience project.

5. The Academy of Medical Royal Colleges (2016) Improving the physical health of adults with severe mental illness: essential actions. OP100 report.
Creative Commons Attribution 4.0 International License

For possible submissions Click Here
Submit Article

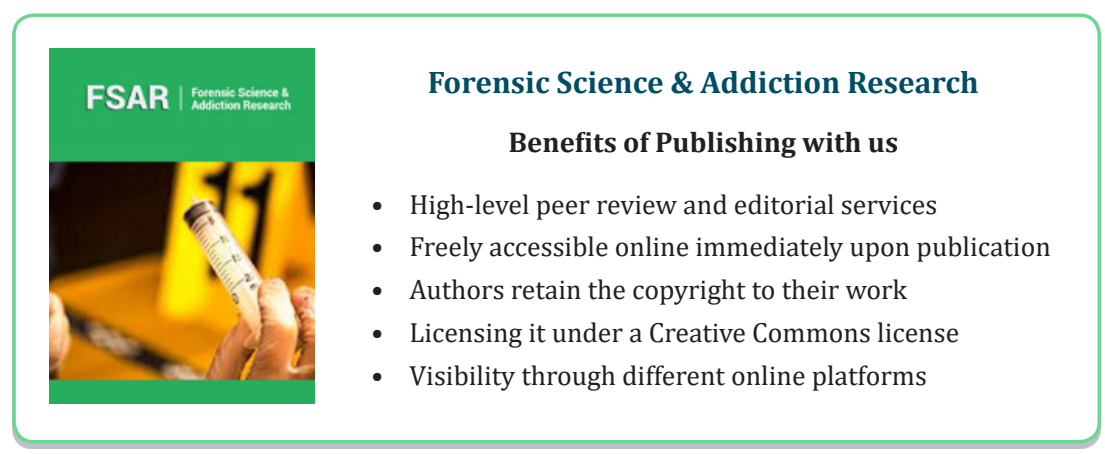

